\title{
Role of flares and shocks in determining solar energetic particle abundances
}

\author{
H. V. Cane, ${ }^{1,2}$ R. A. Mewaldt, ${ }^{3}$ C. M. S. Cohen, ${ }^{3}$ and T. T. von Rosenvinge ${ }^{1}$ \\ Received 13 February 2005; revised 6 February 2006; accepted 15 March 2006; published 23 June 2006.
}

[1] We examine solar energetic particle (SEP) event-averaged abundances of Fe relative to $\mathrm{O}$ and intensity versus time profiles at energies above $25 \mathrm{MeV}$ /nucleon using the SIS instrument on ACE. These data are compared with solar wind conditions during each event and with estimates of the strength of the associated shock based on average travel times to $1 \mathrm{AU}$. We find that the majority of events with an $\mathrm{Fe}$ to $\mathrm{O}$ abundance ratio greater than two times the average 5-12 MeV/nuc value for large SEP events $(0.134)$ occur in the western hemisphere. Furthermore, in most of these Fe-rich events the profiles peak within 12 hours of the associated flare, suggesting that some of the observed interplanetary particles are accelerated in these flares. The vast majority of events with $\mathrm{Fe} / \mathrm{O}$ below 0.134 are influenced by interplanetary shock acceleration. We suggest that variations in elemental composition in SEP events mainly arise from the combination of flare particles and shock acceleration of these particles and/or the ambient medium.

Citation: Cane, H. V., R. A. Mewaldt, C. M. S. Cohen, and T. T. von Rosenvinge (2006), Role of flares and shocks in determining solar energetic particle abundances, J. Geophys. Res., 111, A06S90, doi:10.1029/2005JA011071.

\section{Introduction}

[2] The relative abundances of particle types in solar energetic particle (SEP) events provide important clues about the source populations of the particles and how they were accelerated. It is generally assumed that if the ratios of heavy to light ions are considerably higher than the average value determined by Reames [1998] for large SEP events and/or the relative abundance of ${ }^{3} \mathrm{He}$ to ${ }^{4} \mathrm{He}$ is high compared with the solar wind value, then the ultimate source region of the particles includes plasma that has been heated as a consequence of solar flares. Reames [1998] added together all the $\mathrm{Fe}$ and $\mathrm{O}$ particles counted in 49 large particle events in the range 5-12 MeV/nuc and derived an $\mathrm{Fe} / \mathrm{O}$ ratio of 0.134 . This will be called the Reames value and is close to values determined for the corona [e.g., Fludra and Schmelz, 1999].

[3] Although an $\mathrm{Fe} / \mathrm{O}$ ratio of 10 times the Reames value is quoted as typical of flare particles, it is clear that a range of $\mathrm{Fe} / \mathrm{O}$ values is observed and, for any discussion about the presence or absence of flare particles, one needs to define a lower limit. In a recent paper, Reames and $N g$ [2004] give values that range from 3.3 up to 36 for the $\mathrm{Fe} / \mathrm{O}$ ratio (normalized to the Reames value) in "large impulsive events" (in which only flare particles are expected), and

\footnotetext{
${ }^{1}$ Astroparticle Physics Laboratory, NASA Goddard Space Flight Center, Greenbelt, Maryland, USA.

${ }^{2}$ Also at School of Mathematics and Physics, University of Tasmania, Hobart, Tasmania, Australia.

${ }^{3}$ Space Radiation Laboratory, California Institute of Technology, Pasadena, California, USA.
}

Copyright 2006 by the American Geophysical Union. 0148-0227/06/2005JA011071\$09.00 they note that the heavy-ion enhancement is inversely proportional to the associated flare soft X-ray peak intensity. Reames and $\mathrm{Ng}$ [2004] propose that this result is related to the limited energy available in the smallest flares with the heavy elements absorbing most of the wave energy and therefore being preferentially accelerated. With more energy available a cascade process produces shorter wavelength waves able to resonate with lighter elements and accelerate them, thus resulting in a lowering of the ratio of heavy to lighter ions. This scenario may also explain the earlier result [Reames et al., 1990] that the $\mathrm{Fe} / \mathrm{C}$ ratio is inversely proportional to the flare duration. Reames et al. [1990] found that the $\mathrm{Fe} / \mathrm{C}$ ratio for the Fe-rich events of that study varied from about $0.7-13$, equivalent to an $\mathrm{Fe} / \mathrm{O}$ range of $0.3-6$ or to $2.2-45$ when normalized to the Reames value. On the basis of the results of these two studies it will be assumed in this work that the presence of flare particles can be identified by a normalized $\mathrm{Fe} / \mathrm{O}$ ratio greater than 2.0.

[4] Of current debate is whether the flare-like abundances (i.e., normalized $\mathrm{Fe} / \mathrm{O}$ ratios above 2.0) often seen in large SEP events result directly from the flares that always accompany such SEP events. Since large SEP events are also accompanied by type III- $l$ radio bursts [Cane et al., 2002], indicating the escape of flare electrons to the interplanetary medium, it would be surprising if flare ions did not escape too. Nevertheless many researchers consider that flare particles are unlikely for a number of reasons including timing considerations and the assumed very small source regions [see, e.g., Tylka et al., 2005]. An alternative proposal to the concomitant flare providing flare particles is that, since such events also occur at the times of big, fast coronal mass ejections (CMEs), the CME-driven shocks could have access to a flare particle seed population from 
small flares that occur in the days prior to the large events [see Mason et al., 1999]. In this paper we examine $>25 \mathrm{MeV} /$ nuc $\mathrm{Fe} / \mathrm{O}$ ratios and investigate some of the factors that contribute to the variations in the observed abundances, in particular the presence of shocks in the solar wind during the particle events.

[5] The organization of SEP intensity-time profiles as a function of observer location [Cane et al., 1988] shows that particle acceleration at CME-driven shocks is very important. The east-west asymmetry in profiles has a simple explanation in terms of the Archimedean-spiral form of the interplanetary magnetic field. Western SEP events generally reach peak intensities early after the solar event because of good magnetic connection between the observing spacecraft and particle sources in the low corona on the western hemisphere. In contrast, far eastern events increase slowly and last for many days because connection to the strongest part of a CME-driven shock improves with time and there is little possibility of detecting particles accelerated near the Sun. Central meridian events show intermediate profiles, often with a sudden decrease in intensity about half a day after shock passage when the observer enters the interplanetary extension of a CME (an ICME).

[6] When energetic shocks pass a spacecraft, particle intensities often peak near the shock passage and then show flow directions indicating that their source is the shock. Several studies [van Nes et al., 1984; Sanderson et al., 1985; Tsurutani and Lin, 1985; Cane et al., 1990; Kallenrode, 1996] have shown that shock speed is important. It is usually assumed that diffusive shock acceleration operates whereby particles gain energy by being continually scattered by wave turbulence back and forth across the shock. The escape of a particle from the shock region is determined by its scattering mean free path. Under the assumption that the mean free path is proportional to the particle's rigidity, higher-rigidity particles escape more easily and hence are less efficiently accelerated than lowerrigidity particles. This explains why in situ shock-accelerated populations at energies of the order of $1 \mathrm{MeV} /$ nuc have been observed to have a reduced $\mathrm{Fe} / \mathrm{O}$ ratio and steep spectra [Klecker et al., 1981; Cane et al., 1991, see also Desai et al., 2003]. Since particle events viewed from the west of their solar source region (eastern events) nearly always have an associated shock detected at 1 AU and events viewed from the east tend to be associated with weak shocks or none at all, there is an expectation that $\mathrm{Fe} / \mathrm{O}$ ratios should show some organization with longitude of the associated flare. Indeed this was found in a study of abundances in the $\sim 2 \mathrm{MeV} /$ nuc range for events in solar cycle 21 by Cane et al. [1991]. The events with the lowest $\mathrm{Fe} / \mathrm{O}$ originated near central meridian in association with fast shocks, eastern events had relatively low values, and events with the highest values originated on the western hemisphere. These results confirmed the important role of interplanetary (IP) shock acceleration.

[7] A similar organization by longitude of $14 \mathrm{MeV} / \mathrm{nuc}$ $\mathrm{Fe} / \mathrm{O}$ values was found for the first 27 events of cycle 23 [von Rosenvinge et al., 2001]. Cane et al. [1991] had suggested that events with enhanced heavy ion abundances had a component that came from flare-accelerated particles. Such a component would be observed on field lines that connect to the flaring region. At a distance of $1 \mathrm{AU}$ the
Parker spiral interplanetary magnetic field nominally has footpoints at $\mathrm{W} 60^{\circ}$ but the direct connection varies between about $\mathrm{W} 30^{\circ}$ and $\mathrm{W} 80^{\circ}$ depending on the speed of the solar wind. Furthermore, the longitude of the peak $\mathrm{H} \alpha$ emission may not represent the mean longitude of open field lines which will have some range across the solar disk. Also there is clear evidence that flare particles can occasionally be detected from as far east as E19 ${ }^{\circ}$; Lin [1970] saw impulsive electron increases from this eastern extent and Reames et al. [1988] detected ${ }^{3} \mathrm{He}$ rich events from E12 ${ }^{\circ}$ and E13 ${ }^{\circ}$.

[8] In a recent study, Cane et al. [2003] investigated $\mathrm{Fe} / \mathrm{O}$ ratios in the energy range $25-80 \mathrm{MeV} /$ nuc for 29 events that occurred in the period 1997-2001 with measurable Fe in this energy range. They found that most came from the western hemisphere and had event-averaged $\mathrm{Fe} / \mathrm{O}$ above the Reames value. For the 10 of these events that had lower values there was an IP shock detected at Earth during the particle event. By examining intensity-time profiles, and not just concentrating on event-averaged abundances, Cane et al. [2003] found that a few events showed an Fe-rich component at the start of the event followed by an Fe-poor component at shock passage as might be expected if there is an Fe-rich flare component in large events.

[9] In this paper the study of Cane et al. [2003] is extended to events up to the end of 2005 and includes smaller events selected on the basis of proton intensities. Event-averaged $\mathrm{Fe} / \mathrm{O}$ ratios in the energy range $25-80 \mathrm{MeV} /$ nuc are determined for 80 events with upper limits for six others, and their intensity-time profiles characterized. Intensity-time profiles are examined in conjunction with solar wind data for a number of different events. Most of the $>25 \mathrm{MeV} /$ nuc profiles fall into one of two broad classes; those dominated by rapid onset followed by a gradual decay and those peaking at the passage of the associated IP shock. The former are nearly all Fe-rich and the latter are all Fe-poor.

\section{Data Analysis}

[10] In order to compile a set of large particle events that was unbiased with respect to heavy ions, we started with the NOAA "Solar Proton Events Affecting the Earth Environment" list of 90 increases of protons above $10 \mathrm{MeV}$ (found at http://sec.noaa.gov/ftpdir/indices/SPE.txt) in the years 1997 through December 2005. We determined that three of the NOAA increases were parts of previous events, in four increases there was an additional event, and in three there were two additional events, making a total of 97 events. In addition, the central meridian flare of 17 February 2000 given in the NOAA list did produce a proton event, but the peak intensity above $10 \mathrm{MeV}$ resulted from a separate event on 18 February from behind the west limb as determined from the lack of a soft X-ray flare. The complete list of 97 events is given in Table 1. (Note that there are four events, 8 November 2000, 9 November 2002, 28 May 2003, and 24 July 2005, in which there are likely contributions from additional solar events (as listed) but these contributions cannot be resolved in the particle profiles.) The time given in Table 1 is the maximum of the soft $\mathrm{X}$-ray flare or, in cases of behind the limb events, when the $\mathrm{X}$ rays were occulted, the time is the start of the associated type III-l radio emission [see Cane et al., 2002]. Also given is the flare location and the peak $>10 \mathrm{MeV}$ proton 
Table 1. GOES SEP Events (1997-2005)

\begin{tabular}{|c|c|c|c|c|c|c|c|c|c|c|}
\hline \multirow[b]{2}{*}{ Event $^{\mathrm{a}}$} & \multicolumn{5}{|c|}{ Flare } & \multicolumn{4}{|c|}{ Particles } & \multirow{2}{*}{$\begin{array}{c}\text { Shock } \\
\text { Transit }^{\mathrm{f}} \\
\text { Speed, } \\
\mathrm{km} / \mathrm{s}\end{array}$} \\
\hline & Year & Date & Time $^{b}$ & Location & $\begin{array}{l}\text { X-ray } \\
\text { Peak }\end{array}$ & $\begin{array}{c}>10 \mathrm{MeV} \\
\text { Peak, }{ }^{c} \\
\text { pfu }\end{array}$ & $\begin{array}{c}\text { Summing } \\
\text { Interval }\end{array}$ & $\begin{array}{l}\mathrm{Fe} / \mathrm{O}^{\mathrm{d}} \\
\div 0.134\end{array}$ & Profile $^{e}$ & \\
\hline 1 & 1997 & $4 \mathrm{Nov}$ & 0555 & S14W33 & $\mathrm{X} 2$ & 72 & 4 Nov 04 06-Nov 0609 & $3.11 \pm 0.17$ & $\mathrm{P}$ & 640 \\
\hline 2 & & $6 \mathrm{Nov}$ & 1150 & S18W63 & X9 & 490 & Nov 0612 - Nov 0920 & $6.40 \pm 0.08$ & $\mathrm{P}$ & 500 \\
\hline 3 & 1998 & $20 \mathrm{Apr}$ & 1000 & S43W90 & M1 & 1700 & Apr 20 10-Apr 2412 & $0.032 \pm 0.003$ & $\mathrm{O}$ & 520 \\
\hline 4 & & 2 May & 1335 & S15W15 & $\mathrm{X} 1$ & 150 & May $0214-$ May 0502 & $5.23 \pm 0.22$ & $\mathrm{O}$ & 1120 \\
\hline 5 & & 6 May & 0800 & S11W65 & $\mathrm{X} 2$ & 210 & May 0608 -May 0805 & $3.59 \pm 0.25$ & $\mathrm{P}$ & \\
\hline 6 & & 24 Aug & 2205 & N30E07 & $\mathrm{X} 1$ & 670 & Aug 24 23-Aug 2704 & $0.88 \pm 0.29$ & $\mathrm{~S}$ & 1260 \\
\hline 7 & & $23 \mathrm{Sep}$ & 0700 & N18E09 & M7 & 44 & & & $\mathrm{O}$ & 1030 \\
\hline 8 & & 30 Sep & 1320 & N23W81 & M2 & 1200 & Sep 3013 -Oct 0206 & $2.03 \pm 0.06$ & $\mathrm{O}$ & 1010 \\
\hline 9 & & $5 \mathrm{Nov}$ & 1900 & N26W18 & M9 & 11 & & & $\mathrm{O}$ & 740 \\
\hline 10 & & $14 \mathrm{Nov}$ & 0518 & N28W120 & $\mathrm{C} 1$ & 310 & Nov 14 05-Nov 1716 & $5.02 \pm 0.11$ & $\mathrm{P}$ & \\
\hline 11 & 1999 & $20 \mathrm{Jan}$ & 2004 & N27E95 & M5 & 14 & Jan $2022-$ Jan 2612.5 & $8.6 \pm 1.7$ & $\mathrm{O}$ & PS \\
\hline 12 & & $24 \mathrm{Apr}$ & 1300 & NW150 & $\ldots$ & 32 & Apr 24 14.6-Apr 2621.3 & $1.22 \pm 0.40$ & $\mathrm{P}$ & \\
\hline 13 & & 3 May & 0602 & N15E32 & M4 & 14 & & & $\mathrm{~S}$ & 720 \\
\hline 14 & & 1 Jun & 1930 & NW120 & $\ldots$ & 48 & Jun 1 19-Jun 404 & $4.88 \pm 0.23$ & $\mathrm{P}$ & $\ldots$ \\
\hline 15 & & 4 Jun & 0703 & N17W69 & M3 & 64 & Jun 407 -Jun 604 & $2.86 \pm 0.27$ & $\mathrm{P}$ & $\ldots$ \\
\hline 16 & 2000 & $18 \mathrm{Feb}$ & 0925 & N W120 & $\ldots$ & 13 & Feb 18 10-Feb 2002 & $3.3 \pm 1.5$ & $\mathrm{P}$ & $\ldots$ \\
\hline 17 & & 4 Apr & 1541 & N16W66 & C9 & 55 & Apr 04 16-Apr 0506 & $0.80 \pm 0.19$ & $\mathrm{P}$ & 870 \\
\hline 18 & & 6 Jun & 1525 & N20E18 & $\mathrm{X} 2$ & 84 & Jun $616.1-$ Jun 107.2 & $6.5 \pm 1.5$ & $\mathrm{O}$ & 990 \\
\hline 19 & & 10 Jun & 1702 & N22W38 & M5 & 46 & Jun 10 17-Jun 1203 & $5.86 \pm 0.54$ & $\mathrm{P}$ & PS \\
\hline 20 & & $14 \mathrm{Jul}$ & 1024 & N22W07 & X5 & 24,000 & Jul 14 11-Jul 1819 & $0.62 \pm 0.14$ & $\mathrm{~S}$ & 1600 \\
\hline 21 & & $22 \mathrm{Jul}$ & 1134 & N14W56 & M3 & 17 & $\ldots$ & $<0.82$ & $\mathrm{P}$ & $\ldots$ \\
\hline 22 & & $27 \mathrm{Jul}$ & 1130 & NW120 & $\ldots$ & 18 & $\ldots$ & $<3.7$ & $\mathrm{P}$ & \\
\hline 23 & & 9 Aug & 1525 & N11W11 & $\mathrm{C} 2$ & 17 & & & $\mathrm{O}$ & 805 \\
\hline 24 & & 12 Sep & 1213 & S17W09 & M1 & 320 & Sep 12 12-Sep 1523 & $3.06 \pm 0.31$ & $\mathrm{P}$ & 640 \\
\hline 25 & & 16 Oct & 0728 & N04W95 & M2 & 15 & Oct 1607 -Oct 1915.8 & $4.92 \pm 0.35$ & $\mathrm{P}$ & \\
\hline 26 & & 25 Oct & 1125 & N00W120 & $\mathrm{C} 4$ & 15 & Oct $2513-$ Oct 2721 & $0.98 \pm 0.41$ & $\mathrm{P}$ & PS \\
\hline 27 & & $8 \mathrm{Nov}$ & 2328 & N10W75 & M7 & 14,800 & Nov 823 -Nov 1317 & $0.049 \pm 0.004$ & $\mathrm{O}$ & 1300 \\
\hline $27 \mathrm{~A}$ & & 9 Nov & 1613 & S11E10 & M1 & & & & & 1200 \\
\hline 28 & & $24 \mathrm{Nov}$ & 1455 & N22W07 & $\mathrm{X} 2$ & 100 & Nov $2415-$ Nov 2500 & $2.55 \pm 0.42$ & $\mathrm{P}$ & PS \\
\hline 29 & & $25 \mathrm{Nov}$ & 0131 & N07E50 & M8 & 940 & Nov 25 13.3-Nov 2908 & $0.81 \pm 0.04$ & $\mathrm{~S}$ & 1000 \\
\hline 30 & 2001 & 28 Jan & 1600 & S04W59 & M1 & 49 & Jan $2816-$ Jan 3100 & $4.48 \pm 0.38$ & $\mathrm{P}$ & 630 \\
\hline 31 & & 29 Mar & 1015 & N14W12 & $\mathrm{X} 1$ & 35 & Mar 29 10-Apr 104 & $3.28 \pm 0.22$ & $\mathrm{O}$ & 690 \\
\hline 32 & & $2 \mathrm{Apr}$ & 2151 & N18W82 & $\mathrm{X} 20$ & 1,110 & Apr $222.5-$ Apr 821 & $2.30 \pm 0.04$ & $\mathrm{O}$ & 1020 \\
\hline 33 & & $10 \mathrm{Apr}$ & 0526 & S23W09 & $\mathrm{X} 2$ & 355 & Apr 10 05.5-Apr 1212 & $0.94 \pm 0.04$ & $\mathrm{~S}$ & 1220 \\
\hline 34 & & $12 \mathrm{Apr}$ & 1028 & S19W42 & $\mathrm{X} 2$ & 50 & Apr 12 12-Apr 1400 & $2.11 \pm 0.34$ & $\mathrm{P}$ & \\
\hline 35 & & $15 \mathrm{Apr}$ & 1350 & S20W85 & $\mathrm{X} 14$ & 951 & Apr 15 14-Apr 1800.3 & $5.79 \pm 0.15$ & $\mathrm{P}$ & 700 \\
\hline 36 & & $18 \mathrm{Apr}$ & 0214 & S20W120 & $\mathrm{C} 2$ & 321 & Apr 18 03-Apr 2122 & $3.31 \pm 0.15$ & $\mathrm{P}$ & \\
\hline 37 & & $26 \mathrm{Apr}$ & 1312 & N17W31 & M7 & 57 & & & $\mathrm{~S}$ & 1010 \\
\hline 38 & & 7 May & 0855 & NW140 & $\ldots$ & 30 & May 7 12.5-May 912 & $3.01 \pm 0.48$ & $\mathrm{P}$ & $\ldots$ \\
\hline 39 & & 15 Jun & 1520 & SW130 & $\ldots$ & 26 & Jun 15 16-Jun 1714 & $0.77 \pm 0.49$ & $\mathrm{P}$ & . \\
\hline 40 & & 9 Aug & 1122 & S17E19 & $\mathrm{C} 3$ & 17 & Aug 9 19-Aug 1112 & $<0.35$ & $\mathrm{O}$ & 640 \\
\hline 41 & & 15 Aug & 2355 & W140 & 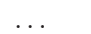 & 493 & Aug 1600 -Aug 2010.7 & $0.90 \pm 0.04$ & $\mathrm{O}$ & PS \\
\hline 42 & & $15 \mathrm{Sep}$ & 1128 & S21W49 & M1 & 11 & & & $\mathrm{P}$ & $\ldots$ \\
\hline 43 & & 24 Sep & 1038 & S16E23 & $\mathrm{X} 2$ & 12,900 & Sep $2411-2.7$ Oct 1 & $0.120 \pm 0.006$ & $\mathrm{~S}$ & 1220 \\
\hline 44 & & $1 \mathrm{Oct}$ & 0515 & S22W91 & M9 & 2,360 & Oct 0107 -Oct 0300 & $0.51 \pm 0.05$ & $\mathrm{O}$ & PS \\
\hline 45 & & 19 Oct & 1630 & N15W29 & $\mathrm{X} 1$ & 11 & Oct 1903.2 -Oct 2120.3 & $2.84 \pm 0.65$ & $\mathrm{P}$ & 870 \\
\hline 46 & & 22 Oct & 1759 & S18E16 & $\mathrm{X} 1$ & 24 & Oct 2217 -Oct 2601 & $5.44 \pm 0.56$ & $\mathrm{P}$ & 640 \\
\hline 47 & & $4 \mathrm{Nov}$ & 1620 & N06W18 & $\mathrm{X} 1$ & 31,700 & Nov 4 16.5-Nov 913.3 & $0.373 \pm 0.008$ & $\mathrm{~S}$ & 1240 \\
\hline 48 & & $17 \mathrm{Nov}$ & 0525 & S13E42 & M2 & 34 & & & $\mathrm{~S}$ & 680 \\
\hline 49 & & $22 \mathrm{Nov}$ & 2330 & S15W34 & M9 & 18,900 & Nov 2223 -Nov 2622.7 & $0.44 \pm 0.02$ & $\mathrm{~S}$ & 1300 \\
\hline 50 & & $26 \mathrm{Dec}$ & 0540 & N08W54 & M7 & 779 & Dec 26 05.5-Dec 2822.7 & $4.94 \pm 0.10$ & $\mathrm{P}$ & 570 \\
\hline 51 & & $28 \mathrm{Dec}$ & 2045 & S26E90 & $\mathrm{X} 3$ & 108 & Dec $2820-\operatorname{Jan} 0300$ & $0.81 \pm 0.08$ & $\mathrm{~S}$ & 870 \\
\hline 52 & 2002 & 8 Jan & 2025 & NE100 & C9 & 91 & $\ldots$ & & $\mathrm{S}$ & 900 \\
\hline 53 & & 14 Jan & 0627 & SW90 & M4 & 15 & & $<2.5$ & $\mathrm{O}$ & $\ldots$ \\
\hline 54 & & $20 \mathrm{Feb}$ & 0612 & N12W72 & M5 & 13 & Feb $2004-F e b 2114$ & $6.9 \pm 1.1$ & $\mathrm{P}$ & \\
\hline 55 & & 15 Mar & 2310 & S08W03 & M2 & 13 & & & $\mathrm{O}$ & 670 \\
\hline 56 & & $18 \mathrm{Mar}$ & 0231 & S09W46 & M1 & 53 & Mar $1806-$ Mar 2009 & $1.43 \pm 0.59$ & $\mathrm{O}$ & 710 \\
\hline 57 & & $22 \mathrm{Mar}$ & 1114 & SW90 & M1 & 16 & & & $\mathrm{~S}$ & 660 \\
\hline 58 & & $17 \mathrm{Apr}$ & 0824 & S14W34 & M2 & 24 & Apr 17 08-Apr 1800 & $1.31 \pm 0.19$ & $\mathrm{P}$ & 860 \\
\hline 59 & & $21 \mathrm{Apr}$ & 0151 & S14W84 & $\mathrm{X} 1$ & 2,520 & Apr $2101.5-$ Apr 2400 & $0.18 \pm 0.007$ & $\mathrm{O}$ & 800 \\
\hline 60 & & 22 May & 0354 & S19W56 & $\mathrm{C} 5$ & 820 & May $2203.5-$ May 2500 & $0.52 \pm 0.05$ & $\mathrm{~S}$ & 1320 \\
\hline 61 & & $7 \mathrm{Jul}$ & 1143 & SW110 & M1 & 22 & Jul 7 12-Jul 920 & $1.81 \pm 0.56$ & $\mathrm{P}$ & \\
\hline 62 & & $15 \mathrm{Jul}$ & 2008 & N19W01 & $\mathrm{X} 3$ & 234 & Jul 1600 -Jul 1708 & $0.73 \pm 0.13$ & $\mathrm{O}$ & 960 \\
\hline 63 & & $20 \mathrm{Jul}$ & 2130 & SE90 & $\mathrm{X} 3$ & 28 & Jul $2100-$ Jul 2512 & $0.59 \pm 0.06$ & $\mathrm{~S}$ & 1300 \\
\hline 64 & & 14 Aug & 0212 & N09W54 & M2 & 26 & Aug 14 01-Aug 1522 & $1.46 \pm 0.42$ & $\mathrm{P}$ & 1400 \\
\hline 65 & & 22 Aug & 0157 & S07W62 & M5 & 36 & Aug $2202-$ Aug 2303 & $5.34 \pm 0.40$ & $\mathrm{P}$ & PS \\
\hline 66 & & 24 Aug & 0112 & S08W81 & $\mathrm{X} 3$ & 317 & Aug $2401-$ Aug 2600 & $5.62 \pm 0.17$ & $\mathrm{P}$ & 710 \\
\hline 67 & & 5 Sep & 1706 & N09E28 & $\mathrm{C} 5$ & 208 & Sep $605-$ Sep 915 & $0.52 \pm 0.17$ & $\mathrm{~S}$ & 880 \\
\hline 68 & & 9 Nov & 1323 & S12W29 & M4 & 404 & Nov 09 14-Nov 1103 & $0.43 \pm 0.12$ & $\mathrm{O}$ & 900 \\
\hline
\end{tabular}


Table 1. (continued)

\begin{tabular}{|c|c|c|c|c|c|c|c|c|c|c|}
\hline \multirow[b]{2}{*}{ Event $^{\mathrm{a}}$} & \multicolumn{5}{|c|}{ Flare } & \multicolumn{4}{|c|}{ Particles } & \multirow{2}{*}{$\begin{array}{c}\text { Shock } \\
\text { Transit } \\
\text { Speed, } \\
\text { km } / \mathrm{s}\end{array}$} \\
\hline & Year & Date & Time $^{\mathrm{b}}$ & Location & $\begin{array}{l}\text { X-ray } \\
\text { Peak } \\
\end{array}$ & $\begin{array}{c}>10 \mathrm{MeV} \\
\text { Peak, }{ }^{\mathrm{c}} \\
\text { pfu }\end{array}$ & $\begin{array}{c}\text { Summing } \\
\text { Interval }\end{array}$ & $\begin{array}{l}\mathrm{Fe} / \mathrm{O}^{\mathrm{d}} \\
\div 0.134\end{array}$ & Profile $^{\mathrm{e}}$ & \\
\hline 69 & 2003 & 28 May & 0027 & S07W17 & $\mathrm{X} 3$ & 121 & May $2802-$ May 3003 & $1.62 \pm 0.15$ & $\mathrm{O}$ & 990 \\
\hline $69 \mathrm{~A}$ & & 29 May & 0105 & S06W37 & $\mathrm{X} 1$ & & & & & 1100 \\
\hline 70 & & 31 May & 0224 & S07W65 & M9 & 27 & May $3103-$ Jun 123 & $3.96 \pm 0.75$ & $P$ & \\
\hline 71 & & 17 Jun & 2255 & S08E61 & M6 & 24 & & & $\mathrm{O}$ & 730 \\
\hline 72 & & 26 Oct & 1819 & N02W38 & $\mathrm{X} 1$ & 466 & Oct 2618 - Oct 2809 & $1.44 \pm 0.05$ & $\mathrm{O}$ & 1300 \\
\hline 73 & & 28 Oct & 1110 & S16E08 & $\mathrm{X} 17$ & 29,500 & Oct 2811 - Oct 2920 & $0.097 \pm 0.007$ & $\mathrm{O}$ & 2210 \\
\hline 74 & & 29 Oct & 2049 & $\mathrm{~S} 15 \mathrm{~W} 02$ & $\mathrm{X} 10$ & 1,570 & Oct $2921-$ Nov 0112 & $1.02 \pm 0.02$ & $\mathrm{O}$ & 2120 \\
\hline 75 & & 2 Nov & 1715 & S20W56 & $\mathrm{X} 8$ & 353 & Nov $0217.7-$ Nov 0420 & $0.69 \pm 0.02$ & $\mathrm{O}$ & 1130 \\
\hline 76 & & $4 \mathrm{Nov}$ & 1929 & S19W83 & $\mathrm{X} 28$ & 100 & Nov 0421 - Nov 0712 & $0.49 \pm 0.03$ & $\mathrm{O}$ & 880 \\
\hline 77 & & 20 Nov & 0747 & N01W08 & $\mathrm{X} 1$ & 13 & Nov $2008-$ Nov 2200 & $1.24 \pm 0.14$ & $\mathrm{P}$ & PS \\
\hline 78 & & $2 \mathrm{Dec}$ & 0948 & S13W65 & $\mathrm{C} 7$ & 86 & Dec $211-\operatorname{Dec} 410$ & $0.87 \pm 0.49$ & $\mathrm{O}$ & \\
\hline 79 & 2004 & $11 \mathrm{Apr}$ & 0419 & S14W47 & C9 & 35 & Apr $1104-$ Apr 122000 & $2.66 \pm 0.31$ & $\mathrm{P}$ & 1100 \\
\hline 80 & & $25 \mathrm{Jul}$ & 1514 & N08W33 & M1 & 2,086 & Jul 25 17.9-Jul 2813 & $0.28 \pm 0.06$ & $\mathrm{~S}$ & 1300 \\
\hline 81 & & $12 \mathrm{Sep}$ & 0056 & N04E42 & M5 & 273 & Sep $1211-$ Sep 1700 & $<0.13$ & $\mathrm{~S}$ & 980 \\
\hline 82 & & 19 Sep & 1712 & N03W58 & M1 & 57 & Sep 19 17.2-Sep 2017.2 & $1.6 \pm 1.1$ & $\mathrm{P}$ & 690 \\
\hline 83 & & $1 \mathrm{Nov}$ & 0650 & W120 & $\ldots$ & 63 & Nov $103.6-$ Nov 216.4 & $1.21 \pm 0.51$ & $\mathrm{P}$ & $\ldots$ \\
\hline 84 & & $7 \mathrm{Nov}$ & 1606 & N09W17 & $\mathrm{X} 2$ & 495 & Nov 7 06.2-Nov 916.2 & $0.49 \pm 0.08$ & $\mathrm{O}$ & 1031 \\
\hline 85 & & $10 \mathrm{Nov}$ & 0213 & N09W49 & $\mathrm{X} 3$ & 300 & Nov $1002-$ Nov 1600 & $1.18 \pm 0.05$ & $\mathrm{O}$ & \\
\hline 86 & 2005 & $15 \mathrm{Jan}$ & 2302 & N15W05 & $\mathrm{X} 3$ & 300 & Jan 15 23-Jan 1709 & $0.28 \pm 0.02$ & $\mathrm{O}$ & 1200 \\
\hline 87 & & 17 Jan & 0952 & N15W25 & $\mathrm{X} 4$ & 4,000 & Jan 1709.5 -Jan 1908 & $0.142 \pm 0.007$ & $\mathrm{O}$ & 1300 \\
\hline 88 & & $20 \mathrm{Jan}$ & 0701 & N14W61 & $\mathrm{X} 7$ & 1,680 & Jan $2007-$ Jan 2200 & $1.65 \pm 0.04$ & $\mathrm{O}$ & 1200 \\
\hline 89 & & 13 May & 1657 & N12E11 & M8 & 3,140 & May 1316.8 -May 1700 & $<3.4$ & $\mathrm{O}$ & 1250 \\
\hline 90 & & 16 Jun & 2002 & N09 W87 & M4 & 44 & Jun 1619.2 -Jun 1814.4 & $2.9 \pm 0.29$ & $\mathrm{P}$ & $\ldots$ \\
\hline 91 & & $13 \mathrm{Jul}$ & 1449 & N10W80 & M5 & 10 & Jul 13 12-Jul 149.6 & $0.56 \pm 0.15$ & $\mathrm{O}$ & $\ldots$ \\
\hline 92 & & $14 \mathrm{Jul}$ & 1055 & N10 W89 & $\mathrm{X} 1$ & 110 & Jul 14 9.6-Jul 1711 & $0.72 \pm 0.04$ & $\mathrm{O}$ & PS \\
\hline 93 & & $17 \mathrm{Jul}$ & 1120 & NW120 & $\ldots$ & 20 & Jul 1711 -Jul 2000 & $1.13 \pm 0.22$ & $\mathrm{P}$ & $\ldots$ \\
\hline 94 & & $24 \mathrm{Jul}$ & 1345 & NE110 & $\cdots$ & 41 & Jul 24 14-Aug 400 & $0.43 \pm 0.06$ & $\mathrm{~S}$ & 530 \\
\hline $94 \mathrm{~A}$ & & $27 \mathrm{Jul}$ & 0502 & N11E90 & M4 & & & & & PS \\
\hline 95 & & 22 Aug & 1727 & S12W60 & M5 & 330 & Aug 22 17-Aug 2600 & $0.43 \pm 0.04$ & $\mathrm{O}$ & 1150 \\
\hline 96 & & $7 \mathrm{Sep}$ & 1740 & S06E89 & $\mathrm{X} 17$ & 1880 & Sep $0717.3-$ Sep 1319 & $0.4 \pm 0.007$ & $\mathrm{~S}$ & 950 \\
\hline 97 & & 13 Sep & 2004 & S09E10 & $\mathrm{X} 1$ & 120 & Sep $1319.2-$ Sep 1600 & $0.25 \pm 0.07$ & $\mathrm{O}$ & 1140 \\
\hline
\end{tabular}

${ }^{a}$ The "A" numbered events are additional solar events that probably produced energetic particles in addition to the main event listed.

${ }^{\mathrm{b}}$ The time is that of soft X-ray maximum intensity or of the start of the type III bursts when the flare was behind the limb.

${ }^{\mathrm{c}}$ Integral intensities from GOES spacecraft. $1 \mathrm{pfu}=1 \mathrm{particle} /\left(\mathrm{cm}^{2} \mathrm{~s}\right.$ ster $)$.

${ }^{\mathrm{d}}$ Normalized $\mathrm{Fe} / \mathrm{O}$ ratios in the range $25-80 \mathrm{MeV} /$ nuc.

"Particle profiles of the type "P" show a prompt onset followed by a slower decay. "S" profiles peak at the time of an associated shock. Most events with an "O" (i.e., other than P or S) profile show a prompt onset but have elevated intensities until the associated shock passes.

"Here "..." indicates no shock near Earth. "PS" indicates there was a passing shock, i.e., a shock not related to the solar event of interest.

intensity. For the beyond the limb events we have estimated longitudes based on the locations of active regions.

[11] We compared this list of large proton events with observations of $>25 \mathrm{MeV} /$ nuc $\mathrm{Fe}$ and $\mathrm{O}$ using the SIS experiment on ACE [Stone et al., 1998]. We specifically chose the highest energy available with reasonable counting statistics because particle acceleration close to the Sun is better probed at high energies where particles accelerated in the interplanetary medium are less likely. For the 80 events with $\mathrm{O}$ and $\mathrm{Fe}$ spectra in the energy range of $25-80 \mathrm{MeV} / \mathrm{nuc}$ that exceeded the background galactic cosmic ray (GCR) level (and, for oxygen, the anomalous cosmic ray (ACR) level) by at least a factor of 2 , we have calculated $\mathrm{Fe} / \mathrm{O}$ ratios. This was done by averaging the measured intensities over the duration of the event, subtracting the appropriate GCR- and ACR-background spectra, and then integrating the resulting time-averaged SEP spectra from 25 to $80 \mathrm{MeV} /$ nucleon. The GCR and ACR background spectra were determined from SIS observations during quiet times near the appropriate SEP event. The time intervals used for averaging each SEP event are given in the table. The uncertainties quoted are statistical only. For six other events, the Fe intensities were not sufficiently above the GCR background level to create well- defined average spectra, although $\mathrm{O}$ spectra were welldefined. For these events we quote upper limits. The remaining events were too small to yield accurate $\mathrm{O}$ spectra.

[12] For all of the GOES events in Table 1 we have classified the intensity-time profiles at energies above 25 $\mathrm{MeV} /$ nuc. For the SIS high-energy events we have examined the $\mathrm{O}$ profiles at $\sim 34 \mathrm{MeV} /$ nuc. For the remaining events we have used GOES $>30 \mathrm{MeV}$ proton intensities. A significant number of events peaked at the passage of the associated shock near $1 \mathrm{AU}$. These events are indicated by an "S" (shock) in the profile column. The four "two component" events discussed by Cane et al. [2003] are in this group. Also included in this group are the far eastern events in which the particles peaked after shock passage. There were a total of 20 "S" events. About half of the events showed a rapid increase close to the time of the associated flare (peaking within 12 hours) followed by a slower decay; such profiles are indicated with a "P" (for prompt) in the profile column. There were 39 "P" events. Excluded from this group were events that had a prompt onset but in which the $\sim 34 \mathrm{MeV} /$ nuc $\mathrm{O}$ was still above background when the associated shock passed the spacecraft; in many cases there was a small enhancement at the 



YEAR

Figure 1. Event-averaged values of $\mathrm{Fe} / \mathrm{O}$ in the range $25-80 \mathrm{MeV} / \mathrm{nuc}$ (normalized to the Reames value) plotted versus time of the associated solar flare event are shown in the lower panel. The gray shading indicates the period of greatest sunspot activity. The upper panel shows the percentage of events that were Fe-rich in three time periods. Also shown is the percentage of associated shocks that had transit speeds greater than $1000 \mathrm{~km} / \mathrm{s}$.

time of shock passage. These events, and all others that could not be classed as $\mathrm{S}$ or $\mathrm{P}$, were grouped together as having "O" (other) profiles. There were 38 "O" events.

[13] In order to investigate whether abundance variations are related to solar wind conditions during the events the passages of shocks and ICMEs were obtained by examining data from the MAG [Smith et al., 1998] and SWEPAM [McComas et al., 1998] experiments, also on ACE. ICME time periods prior to 2003 have been listed by Cane and Richardson [2003] along with probable CME associations and the implied shock transit times from the Sun to $1 \mathrm{AU}$. This list has been updated until the present time (I. G. Richardson and H. V. Cane, manuscript in preparation,
2006). In a number of events an unrelated shock passed during the particle increase. These 10 cases are designated "PS" (passing shock) in the shock transit speed column. The transit speeds give some idea about which shocks are likely to be efficient in particle acceleration. Because fast shocks decelerate between the Sun and 1 AU transit speeds are often better indicators of this efficiency than in situ speeds.

[14] The distribution of the $\mathrm{Fe} / \mathrm{O}$ ratios (normalized by the Reames value of 0.134 ) for the 86 events as a function of time is shown in Figure 1 (lower panel). The solid horizontal line indicates a normalized $\mathrm{Fe} / \mathrm{O}$ of 2.0 and points above this line indicate events with an average 


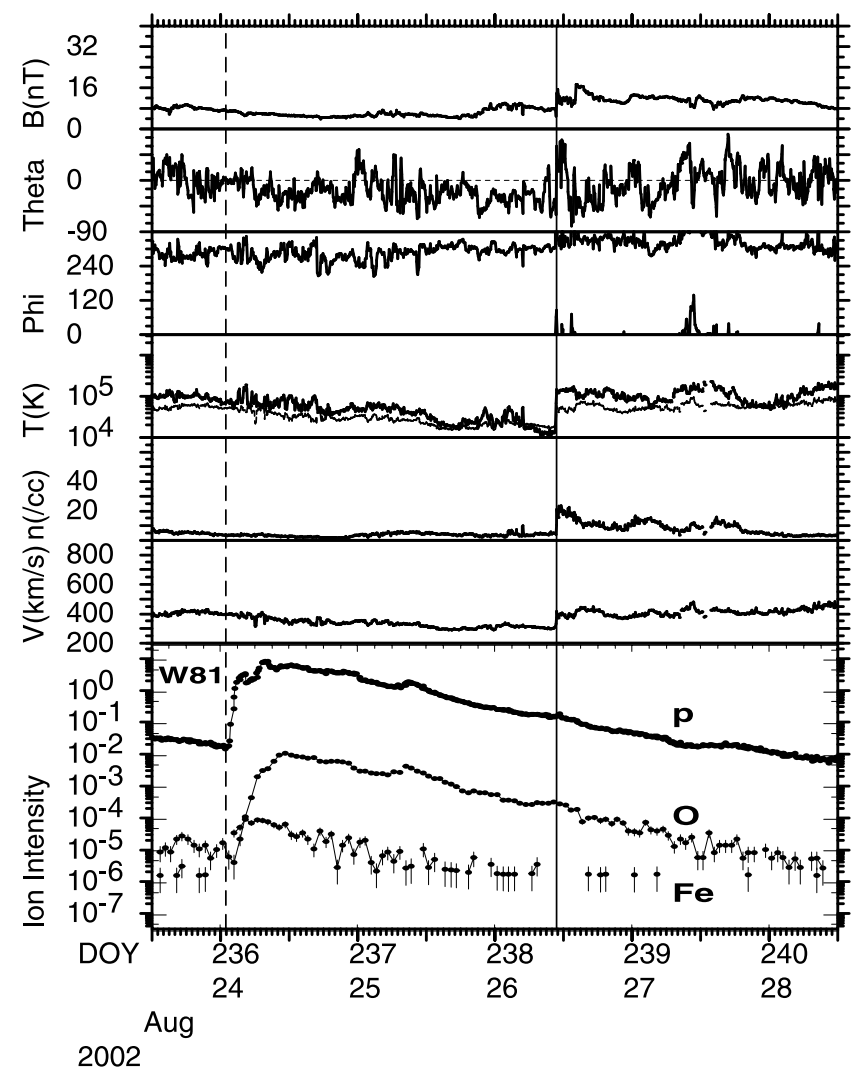

Figure 2. Solar wind data combined with particle data for an event with a "prompt" profile. The top three panels show magnetic field strength and its direction. The next three panels show the solar wind temperature, density, and speed. The two curves in the temperature panel are the observed temperature and the temperature expected on the basis of the solar wind speed. The dashed vertical line indicates the time of the associated flare and the solid line indicates the passage of the associated shock. The particle profiles in the bottom panel are intensities of (in decreasing order) 19-28 MeV protons, 7-10 MeV/nuc O, and 22$27 \mathrm{MeV} /$ nuc Fe. The units are particles/( $\mathrm{cm}^{2} \mathrm{sr} \mathrm{s} \mathrm{MeV/nuc)}$. The Fe event is essentially at background level by the time the shock passes.

composition that is assumed to include a significant contribution from flare particles. The dashed horizontal line indicates a normalized $\mathrm{Fe} / \mathrm{O}$ of 1.0 and points well below this line indicate events with an average composition that is below the typical coronal value, consistent with rigidity related effects of diffusive shock acceleration as discussed in section 1 . The grey shading indicates the period when the smoothed sunspot number was above $75 \%$ of the maximum of cycle 23. Figure 1 (upper panel) shows the percentage of Fe-rich events in three intervals, before, during, and after maximum solar activity. The upper panel also shows the percentage of associated shocks that had transit speeds above $1000 \mathrm{~km} / \mathrm{s}$. (That there are more fast CMEs and fast interplanetary shocks after solar maximum has been reported previously [Cane et al., 1996]).

[15] Figure 2 shows particle and solar wind data for an event that occurred on 24 August 2002. The particle data are
19-28 MeV protons, 7-10 MeV/nuc O and 22-27 Mev/ nuc Fe. The proton data are from the EPACT experiment on Wind [von Rosenvinge et al., 1995] and the $\mathrm{O}$ and $\mathrm{Fe}$ are from the SIS experiment. The dashed line indicates the time of the associated flare at $\mathrm{W} 81^{\circ}$ and the solid line the passage of the associated shock at $1 \mathrm{AU}$. The jump of about $100 \mathrm{~km} / \mathrm{s}$ in the solar wind speed $(\Delta \mathrm{V})$ at shock passage indicates that the shock was of moderate strength. Nevertheless, there was little effect on the energetic particle profiles that peaked within hours of the flare. This is an example of a "prompt" event. (Note that the term "shock strength" is usually taken to mean the shock compression as given by the ratio of the densities across the shock; Feldman et al. [1983] showed that an equivalent measure is the difference in bulk velocity across the shock.)

[16] Figure 3 shows particle and solar wind data for a "shock" event that occurred on 5 September 2002. The flare was at E28 $8^{\circ}$ The particles peaked at the time of the passage of the strong shock $(\Delta \mathrm{V} \sim 200 \mathrm{~km} / \mathrm{s})$. The gray shading indicates the times during which ICMEs passed 1 AU. Figure 4 shows data for a shock event that did not have an increase in the Fe intensity above $25 \mathrm{MeV} /$ nuc and originated just beyond the east limb on 8 January 2002. This event is shown because it illustrates the rise in intensity after shock passage that is usually seen for far eastern events [cf. Cane et al., 1988, Figure 7].

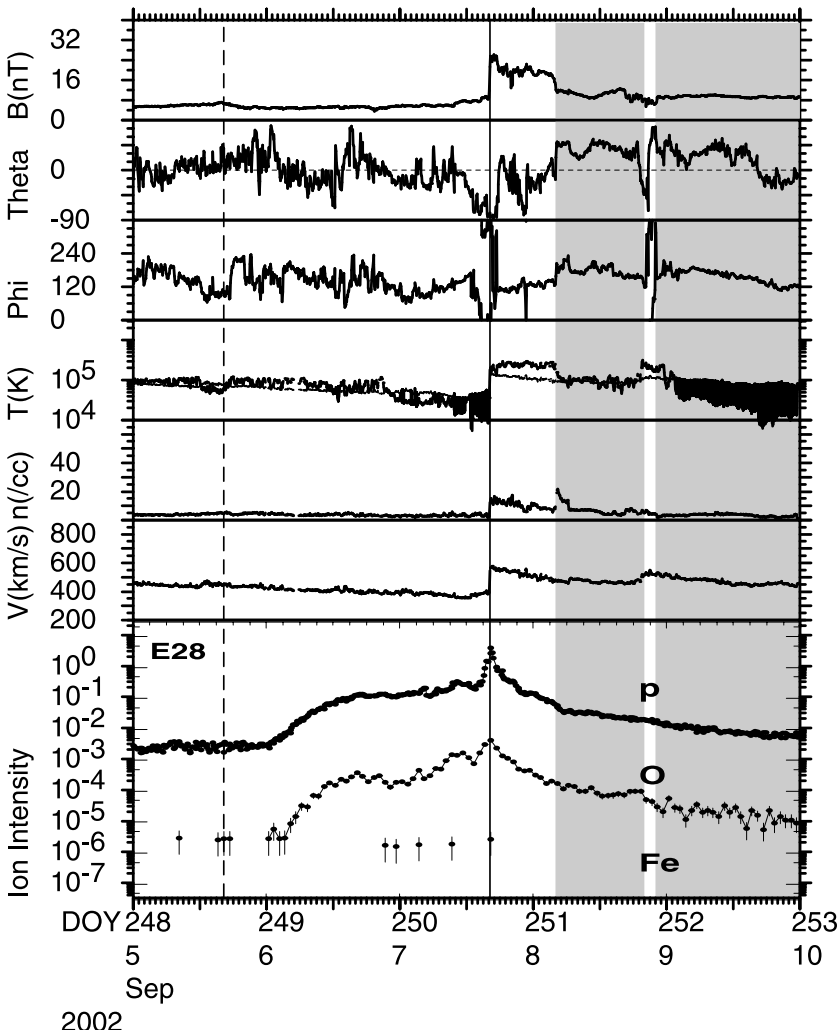

Figure 3. Solar wind and particle data for an event in which the profiles peak at shock passage. The gray shading indicates times when the spacecraft was inside an ICME. The black area in the temperature panel indicates a region of lower then expected temperature and is usually indicative of the presence of an ICME. 


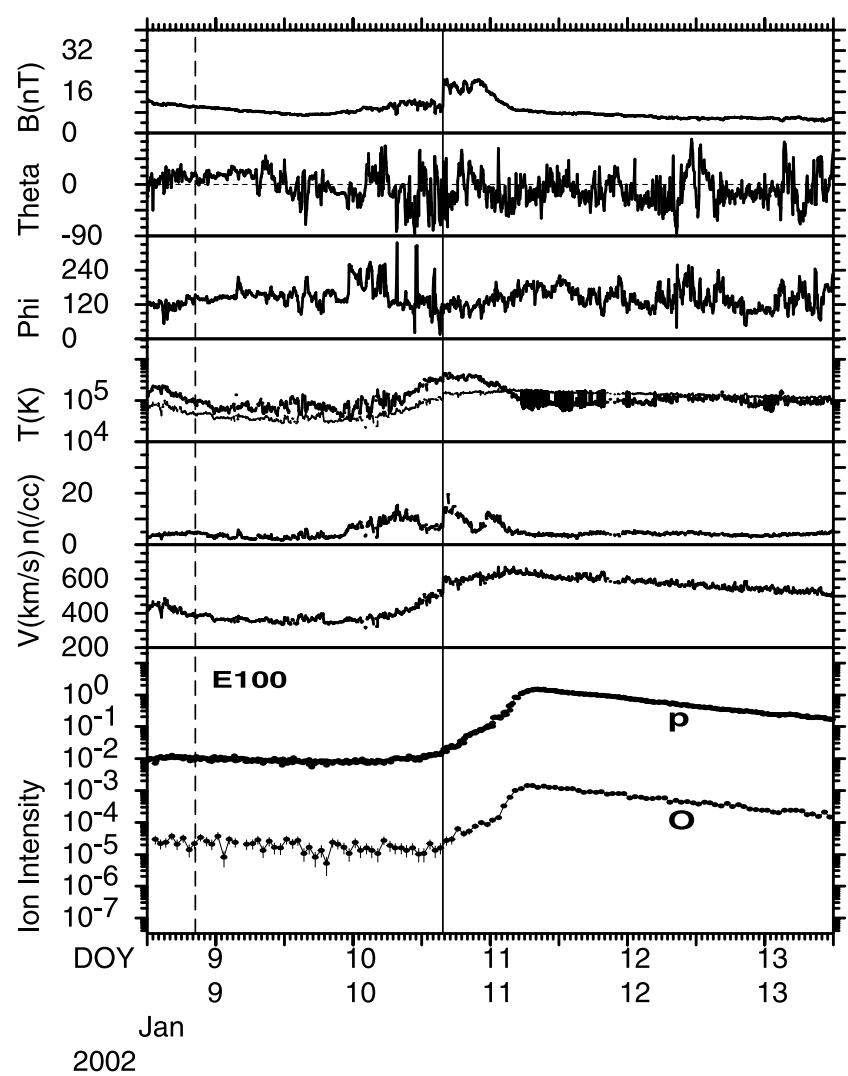

Figure 4. Solar wind and particle data for an event from behind the east limb in which the profiles peak after shock passage.

[17] Most of the SEP events with "other" profiles had relatively strong shocks that kept intensities high until after the shocks passed. Some have complicated profiles because of the presence of other shocks and ICMEs, i.e., ones that did not originate in the same solar event as the energetic particles. Figure 5 shows data for an event like this that occurred 1 October 2001 and originated just behind the west limb. The soft X-ray flare reached maximum at $0515 \mathrm{UT}$. The profiles are $\mathrm{O}$ at $21-29$ and $29-39 \mathrm{MeV} / \mathrm{nuc}$ and $\mathrm{Fe}$ at 26-36 MeV/nuc and it can be seen that they are not prompt. The upper points show Fe/O at $34 \mathrm{MeV} /$ nuc. It can be seen that the ratio is about 2 at the beginning of the event but decreases near the time of the vertical line which marks the time of a probable shock. Later in the event the O profiles attain peak intensity inside an ICME; by that time there is little high-energy Fe. Thus the event-averaged, normalized $\mathrm{Fe} / \mathrm{O}$ is $<1$.

[18] Figure 6 shows the distribution of the event characteristics (profile type and average normalized $\mathrm{Fe} / \mathrm{O}$ ratio) as a function of the longitude of the associated flare for the 86 events. Note that there are three eastern events (20 January 1999, 6 June 2000, and 22 October 2001) with $\mathrm{Fe} / \mathrm{O}>2.0$ and one other eastern event (13 May 2005) with an upper limit above 2.0. In contrast, 13 eastern events had $\mathrm{Fe} / \mathrm{O}<1$, including seven with $\mathrm{Fe} / \mathrm{O}<0.5$. There are also two western events (20 April 1998 and 8 November 2000) with very low Fe/O ratios. For the 20 April 1998 event the intensity profiles were unusual for an event from the west limb. Instead of the $\sim 25 \mathrm{MeV} /$ nuc Fe profile peaking within about 6 hours of the flare, as in the event shown in Figure 2, the profile at this energy, and also those at lower energies, peaked about a day after the flare as may be seen in Figure 7. The 8 November 2000 particle event was likely the result of two solar events both including strong shocks. The second solar event on 9 November originating near central meridian, produced a strong type III- $l$ burst and strong interplanetary shock radio emissions (see http:// lep694.gsfc.nasa.gov/waves/waves.html).

[19] The results for the 80 events with finite values may be summarized as follows. (Note again that all the $\mathrm{Fe} / \mathrm{O}$ ratios are normalized to the Reames value of $\mathrm{Fe} / \mathrm{O}=0.134$.) For those events with prompt profiles similar to those shown in Figure 1 (36 events with finite values for Fe/O), the event-averaged $\mathrm{Fe} / \mathrm{O}$ ratios were mostly consistent with flare composition and all but one originated in the western hemisphere. Sixty-nine percent $(25 / 36)$ had $\mathrm{Fe} / \mathrm{O}>2.0$. It may be significant that 6 of the 11 with $\mathrm{Fe} / \mathrm{O}<2.0$ originated from behind the west limb where magnetic connection to Earth is generally poor. For the 20 events with peak intensities at shock passage we could determine a high-energy $\mathrm{Fe} / \mathrm{O}$ ratio for 14 of them; none had $\mathrm{Fe} / \mathrm{O}>1.0$.

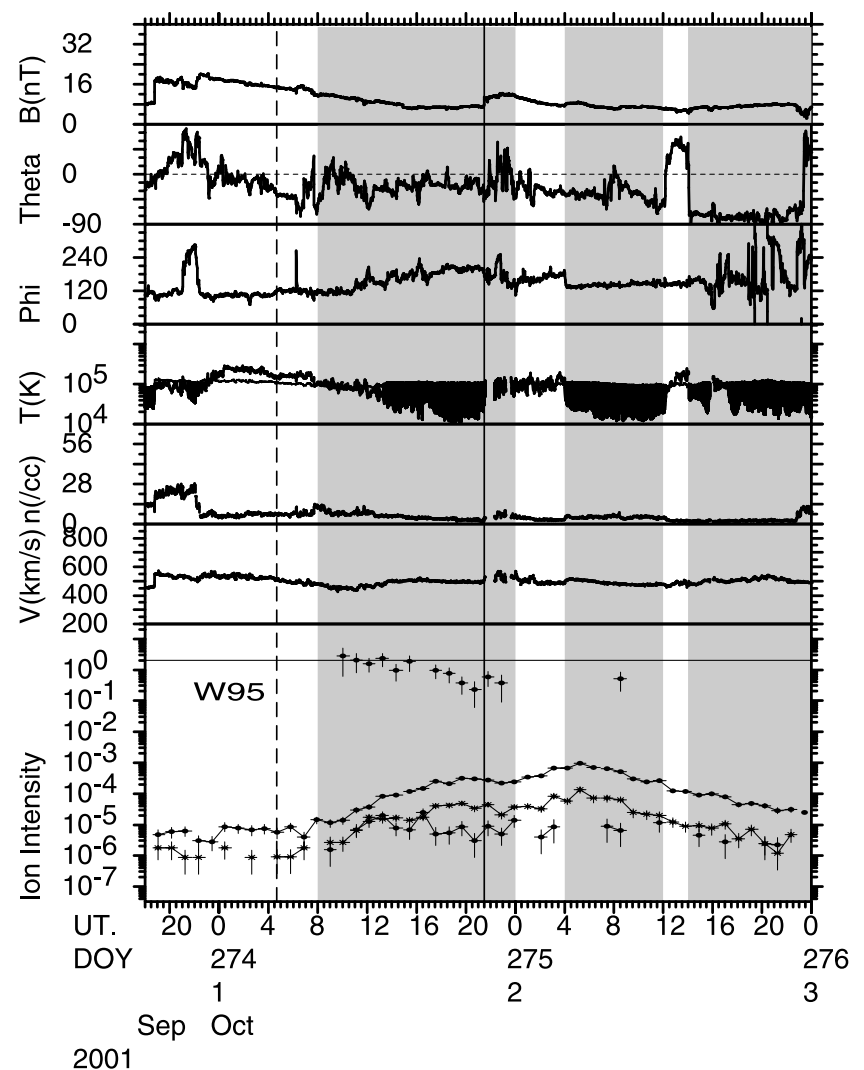

Figure 5. Solar wind and particle data for an event in which the solar wind was complicated and affected the particle profiles. The profiles are at $21-29$ and 29$39 \mathrm{MeV} /$ nuc $\mathrm{O}$ and $\mathrm{Fe}$ at $26-36 \mathrm{MeV} /$ nuc. The gray shading shows the times of several ICMEs that appear to control the $\mathrm{O}$ intensities. The solid line is at the time of a possible shock. The highest line of particle data in the bottom panel is the $\mathrm{Fe} / \mathrm{O}$ ratio at $34 \mathrm{MeV} /$ nuc; the horizontal line shows where $\mathrm{Fe} / \mathrm{O}$ equals 2.0. 


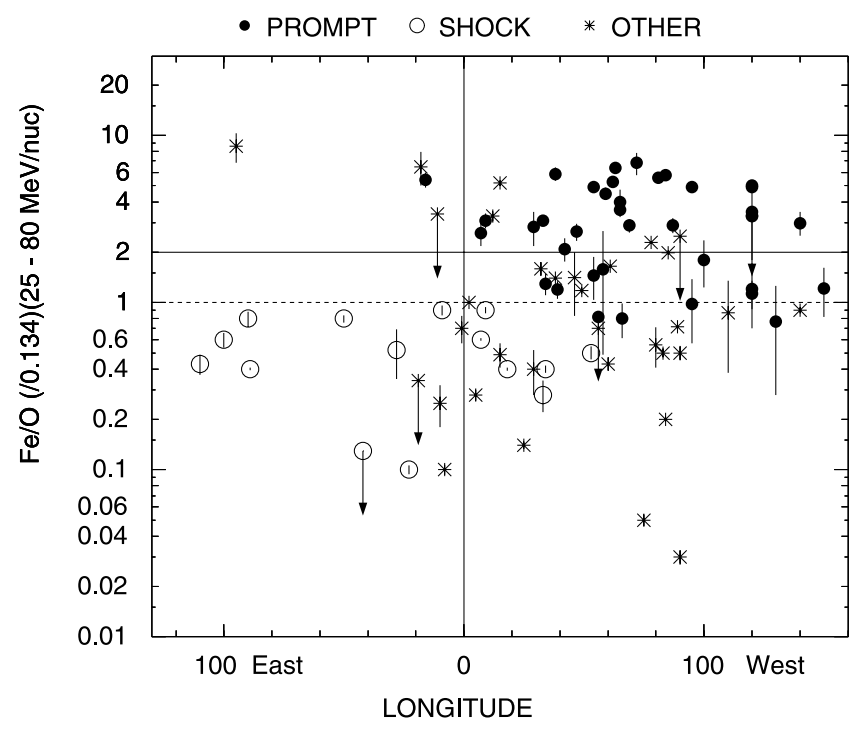

Figure 6. Event-averaged values of $\mathrm{Fe} / \mathrm{O}$ in the range 25-80 MeV/nuc (normalized to the Reames value) plotted versus longitude of the associated solar flare event. The symbols indicate the profile type; filled circles represent the prompt events, unfilled circles represent the shock events, and asterisks are used for the events with "other" profiles.

The remaining events had profiles that were not prompt, as defined above, nor did they peak at the passage of the associated shock; more than half $(63 \%)$ had $\mathrm{Fe} / \mathrm{O}<1.0$.

[20] The 11 NOAA proton events that did not have measurable heavy ions above $25 \mathrm{MeV} /$ nuc were reasonably small events with the largest being that of 8 January 2002 (illustrated in Figure 4). The event consisted of only interplanetary shock accelerated particles. SIS did detect $\mathrm{O}$ above $12 \mathrm{MeV} /$ nuc giving an upper limit $\mathrm{Fe} / \mathrm{O}$ (normalized) of 0.02 in the range $12-25 \mathrm{MeV} /$ nuc. Four other of these small events with no $\mathrm{Fe}>25 \mathrm{MeV} /$ nuc had profiles indicating that the particles were accelerated in the interplanetary medium.

[21] The complexity of abundance variations is exemplified by the fact that there were nine small NOAA proton events (peak intensity of $>10 \mathrm{MeV}$ protons $<20$ particles/ $\left(\mathrm{cm}^{2} \mathrm{~s}\right.$ ster)) that had measurable Fe above $25 \mathrm{MeV} /$ nuc. One of these occurred on 20 January 1999. Figure 8 shows data (19-28 MeV protons, 7-10 MeV/nuc O, and 22$27 \mathrm{Mev} / \mathrm{nuc} \mathrm{Fe}$ ) for this period. This far eastern event was unusual because the particles started rather abruptly within a few hours of the flare. There was a short duration enhancement on 23 January after the passage of a relatively weak shock, followed by an ICME; the profiles look similar to those of many western events and unlike those of the more typical eastern event shown in Figure 4. It is very unlikely that the 22 January 1999 shock and following ICME originated with the solar event from the east limb on 20 January because this would imply that the ICME had an angular extent of more than $90^{\circ}$, considerably larger than the sizes implied from multispacecraft observations of cosmic ray decreases that are caused by ICMEs [Cane et al., 1994]. It is remarkable that this event had the highest $\mathrm{Fe} / \mathrm{O}$ ratio of the complete set of NOAA proton events. The normalized value of $8.6 \pm 1.7$ is close to that quoted for socalled flare/"impulsive" events. There was only one impulsive event in the NOAA proton list, 20 February 2002, and it had a normalized $25-80 \mathrm{MeV} / \mathrm{nuc} \mathrm{Fe} / \mathrm{O}$ ratio of $6.9 \pm 1.1$.

\subsection{Variations With Energy}

[22] Figure 9 shows the event-averaged 25-80 MeV/nuc $\mathrm{Fe} / \mathrm{O}$ ratios divided by those determined by Reames and $\mathrm{Ng}$ [2004] in the energy range $3-10 \mathrm{MeV} / \mathrm{nuc}$ as a function of the longitude of the associated flare. In the figure the symbols represent the transit speeds of the associated shocks as given in the last column of Table 1. The filled squares are used for events in which the shocks had transit speeds above $1000 \mathrm{~km} / \mathrm{s}$. The solid line shows where

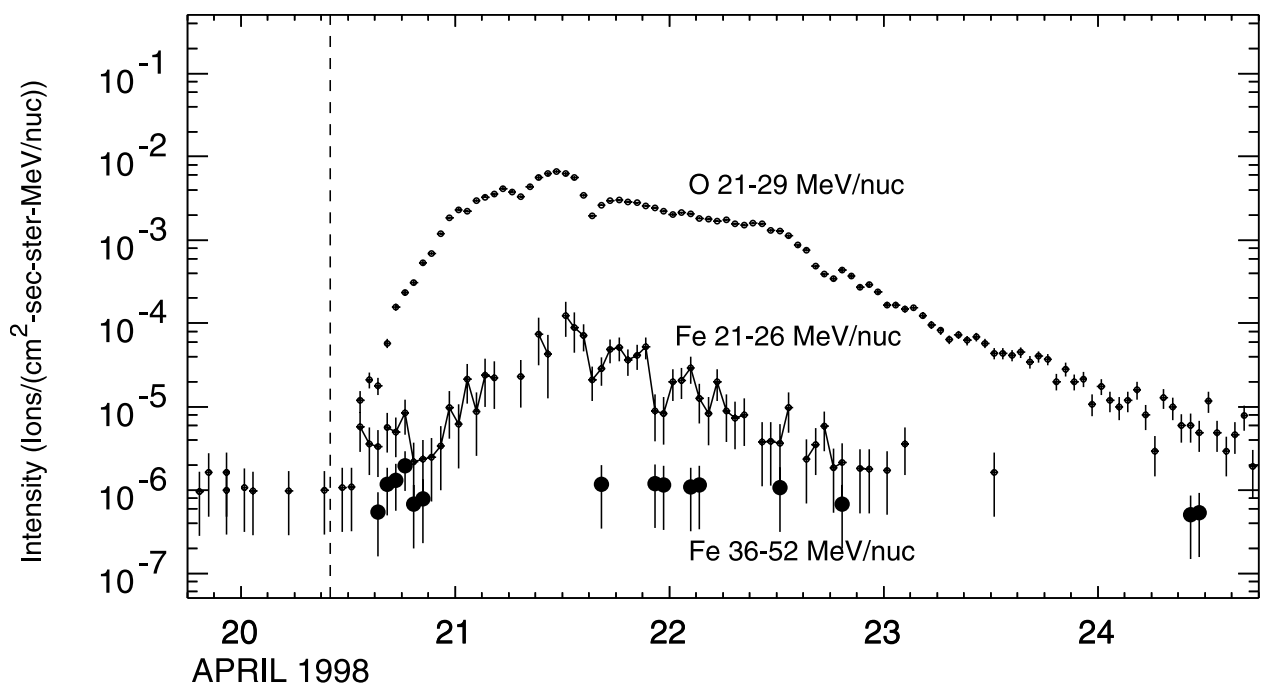

Figure 7. Intensity-time profiles for the unusual event of 20 April 1998. The profiles are O $21-$ $29 \mathrm{MeV} /$ nuc and Fe at 21-26 and 36-52 MeV/nuc. Note the short duration Fe-rich component at the start of the event. The dashed line indicates the time of the flare. 




Figure 8. Solar wind and particle data for a far eastern event with an unusual profile for a supposedly poorly connected event. The intensity profiles are $19-28 \mathrm{MeV}$ protons, 7-10 MeV/nuc O, and 22-27 MeV/nuc Fe. The dashed line is at the time of the associated flare and the solid line indicates the time of a passing shock.

the points would lie if the abundances were not energy dependent.

[23] The western events with low ratios are 20 April 1998 and 8 November 2000; they had flare-like abundances at low energies but very low $\mathrm{Fe} / \mathrm{O}$ values at high energies. The third western event with a relatively low ratio was 20 April 2002. For the vast majority of the remaining western events the $\mathrm{Fe} / \mathrm{O}$ ratio is higher in the high-energy range (the ratio of ratios is greater than 1). The two events with the highest ratios of ratios (6 June 2000 and 12 September 2000) had flare-like abundances at high energies but very low $\mathrm{Fe} / \mathrm{O}$ values at low energies. For central meridian events the highenergy ratio can be considerably higher as well as lower than the low-energy ratio. In contrast, for the two eastern events the ratios are about the same. Note that these two events are both from near the east limb and the particles are not locally accelerated but are a population well behind the shock that is probably trapped there. There are four other eastern events for which Reames and $\mathrm{Ng}$ [2004] provide a ratio and all were less than 1 . These events had so little high-energy Fe that we could not determine a ratio.

[24] Note that there are only a few events in Figure 9 without associated shocks (the small filled circles). This is because the Reames and Ng [2004] events, being at a lower energy than ours and selected for a higher peak intensity, are dominated by locally shock accelerated populations. Seven of the nine events that are on the Reames and $N g$ [2004] list but for which there are insufficient high-energy ions to determine the ratio at high energies peaked at shock passage. In the next section we suggest a possible explanation for the energy variations.

\section{Discussion and Summary}

[25] This study finds that when particles peak close to the time of the associated flare the normalized $25-80 \mathrm{MeV} / \mathrm{nuc}$ $\mathrm{Fe} / \mathrm{O}$ ratio is generally greater than 2.0 and therefore consistent with the range of abundance ratios found in smaller flare events at lower energies. For events that peak near the passage of the associated shock or have enhancements centered on shock passages, the ratio is generally less than 1.0 and consistent with the less efficient shock acceleration of $\mathrm{Fe}$. This is consistent with solar energetic particle events having two components; an Fe-rich component that originates in flares and a shock component that is usually Fe-poor.

[26] Whereas the presence of flare particles in large SEP events is now widely accepted, it is often assumed that these flare particles are shock accelerated remnants from previous small flares [Mason et al., 1999; Tylka et al., 2005]; Reames [2002] has proposed that in large events there are no open field lines along which flare particles can escape from the low corona. However this is unlikely to be correct because of the observed type III radio emissions [Cane et al., 2002] indicating that flare-accelerated electrons have access to the interplanetary medium. We therefore think it much more likely that the flare particles originate in the concomitant flare. The extent to which these flare particles are further accelerated by the associated shock is unclear but this will

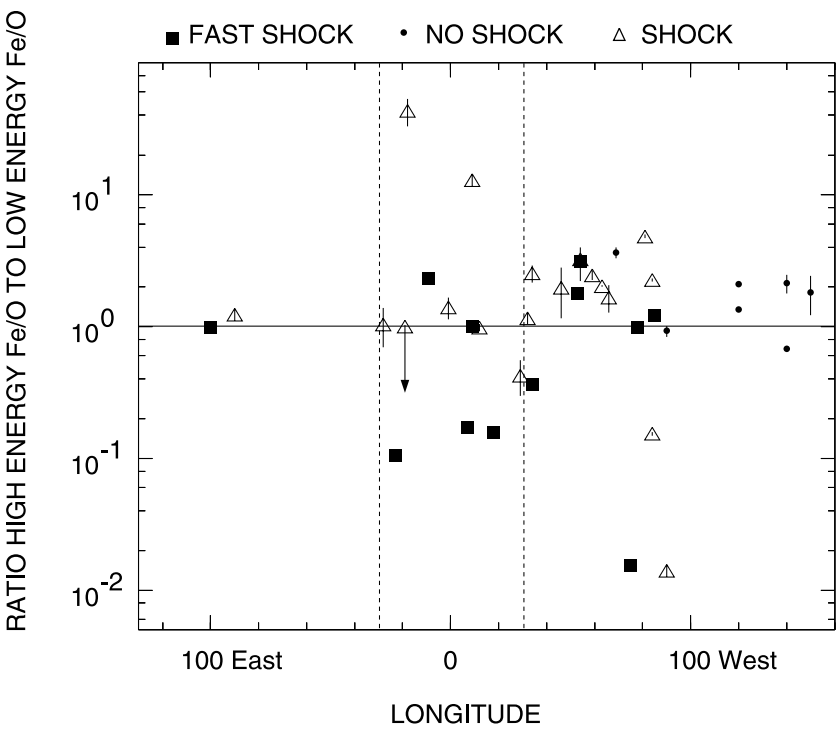

Figure 9. Ratio of event-averaged $25-80 \mathrm{MeV} / \mathrm{nuc} \mathrm{Fe} / \mathrm{O}$ to the $3-10 \mathrm{MeV} /$ nuc $\mathrm{Fe} / \mathrm{O}$ plotted versus the longitude of the associated flare. Symbols indicate whether there was a shock at $1 \mathrm{AU}$ and whether the transit speed was below $1000 \mathrm{~km} / \mathrm{s}$ or not. The horizontal line indicates equal abundances in the two energy ranges. The dashed vertical lines delineate central meridian longitudes. 
certainly depend on the energy and species of the particles and the shock properties.

[27] It is important to remember that the abundances quoted in this paper, and most others, are event averages. Thus when comparing average abundances at different energies the comparison is often between particles accelerated predominantly in the interplanetary medium (lowenergy measurements) with particles typically accelerated close to the Sun (high-energy measurements). Even when both measurements are of interplanetary populations one might expect differences because the rigidity effects in shock acceleration that lead to lowered $\mathrm{Fe} / \mathrm{O}$ ratios only occur above some energy dependent on the characteristics of the shock.

[28] It has been suggested that high $\mathrm{Fe} / \mathrm{O}$ in large events arises because of selective acceleration of flare particles by a perpendicular shock [Tylka et al., 2005]. We see no need to introduce the additional variable of shock geometry into our proposed two component (flare and interplanetary shock) scenario. We propose that event to event variations result from variations in the intensity, abundances, and angular extent of the flare population and variations in the strength and angular extent of the shock. For individual events these properties will depend on the location of the observer. Further variations can result from additional flares, additional shocks in the interplanetary medium and because field lines may not follow a Parker spiral. The latter situation can occur when a spacecraft is inside an ICME. Examples of eastern events that are apparently well connected to near-Earth spacecraft via ICMEs have been reported [Richardson et al., 1991; Richardson and Cane, 1996]. The presence of an ICME might explain the near eastern event of 22 October 2001 that had $\mathrm{Fe} / \mathrm{O}$ above 2.0. On the other hand, as noted in section 1, flare particles have been seen in small events originating as far east as E19 indicating that there are other field line geometries (besides ICMEs) allowing flare particles to reach the Earth from just east of central meridian. Since all but one of the events of this study with flare-like abundances originate within this eastern boundary, particles from the concomitant flare seem a probable source of flare particles in large events.

[29] We have no explanation for the high $\mathrm{Fe} / \mathrm{O}$ obtained for the far eastern event on 20 January 1999 but note again that the event had a prompt onset shortly after the associated flare. The standard explanation for good connection to particles accelerated very soon after the start of a solar event is that the CME shock extended to the foot of the field line near Earth. However, the observations suggest that the shock in the January 1999 event was not particularly energetic. The shock was probably not detected at Earth and the associated radio emissions did not extend below $2 \mathrm{MHz}$ (when the shock was still within $0.03 \mathrm{AU}$ of the Sun). In contrast, the shock associated with the more typical far eastern event of January 2002 (illustrated in Figure 4) generated radio emissions all the way to where it was detected near Earth.

[30] For events originating near central meridian we find that the event-averaged $\mathrm{Fe} / \mathrm{O}$ ratios at $\sim 30 \mathrm{MeV} /$ nuc can be higher or lower than those at $\sim 5 \mathrm{MeV} /$ nuc. In our scenario this is a consequence of both flare and shock components contributing to observations near Earth. Events with a high ratio at high energies but a low one at low energies could occur because the event is dominated by shock accelerated particles at low energies but, when the shock is not too strong, a flare contribution becomes more dominant at high energies. Events with a low $\mathrm{Fe} / \mathrm{O}$ ratio at $\sim 30 \mathrm{MeV} /$ nuc and a high value at $\sim 5 \mathrm{MeV} /$ nuc could arise because a very strong shock efficiently accelerates a flare population at low energies (thus not changing the $\mathrm{Fe} / \mathrm{O}$ ratio of the source by much) but is not as efficient at high energies resulting in a reduced $\mathrm{Fe} / \mathrm{O}$. Some support for this suggestion is the observation that the events with these energy dependencies in $\mathrm{Fe} / \mathrm{O}$ tend to be the ones with the high transit speed shocks as shown in Figure 9.

[31] The topic of classes of SEP events will be discussed elsewhere (H. V. Cane, manuscript in preparation, 2006); however it is relevant to note that the suggestion that the flare particles in large events originate in the concomitant flare implies then that the only differences between small and large SEP events is the absence/presence of an interplanetary shock and the difference in the size and conditions (e.g., density) of the flaring region. Support for this suggestion is that the more intense flare particle events have associated CMEs, albeit smaller ones than for the largest SEP events. However, the 20 February 2002 SEP event was accompanied by a halo CME as is the case in the majority of large SEP events. In our scenario the high $\mathrm{Fe} / \mathrm{O}$ in this event is attributed to the impulsive flare and the absence of an interplanetary shock (there is no observational evidence for an interplanetary shock).

[32] In conclusion we have determined event averaged $\mathrm{Fe} / \mathrm{O}$ ratios in the energy range $25-80 \mathrm{MeV} /$ nuc for all large SEP events for solar cycle 23. We find that the abundances are reasonably well organized by event profiles such that events reaching a peak intensity shortly after the associated flare are Fe-rich and events peaking at the passage of an interplanetary shock are Fe-poor. We suggest that variations in elemental composition in SEP events arise mainly from the combination of flare particles from the associated flare and shock acceleration of these particles and/or the ambient medium.

[33] We anticipate that for most events observed by the upcoming multispacecraft STEREO mission the wellconnected spacecraft will measure a higher $\mathrm{Fe} / \mathrm{O}$ ratio than the other spacecraft. Closer to the Sun, the flare and shock components should be much better defined. NASA's planned Inner Heliospheric Sentinels mission could provide definitive tests of this model with solar-wind data and SEP composition, spectra, and timing measurements from four spacecraft distributed in longitude and radius between 0.25 and $0.7 \mathrm{AU}$ from the Sun.

[34] Acknowledgments. H.V.C. was supported at GSFC by a contract with USRA. The work at California Institute of Technology was supported by NASA under grant NAG5-12929. We thank the referees for constructive criticism.

[35] Shadia Rifai Habbal thanks Stephen Kahler and the other two referees for their assistance in evaluating this paper.

\section{References}

Cane, H. V., and I. G. Richardson (2003), Interplanetary coronal mass ejections in the near-Earth solar wind during 1996-2002, J. Geophys. Res., 108(A4), 1156, doi:10.1029/2002JA009817.

Cane, H. V., D. V. Reames, and T. T. von Rosenvinge (1988), The role of interplanetary shocks in the longitude distribution of solar energetic particles, J. Geophys. Res., 93, 9555. 
Cane, H. V., T. T. von Rosenvinge, and R. E. McGuire (1990), Energetic particle observations of CME-associated shocks at the Helios-1 spacecraft, J. Geophys. Res., 95, 6575.

Cane, H. V., D. V. Reames, and T. T. von Rosenvinge (1991), Solar particle abundances at energies greater than $1 \mathrm{MeV}$ per nucleon and the role of interplanetary shocks, Astrophys. J., 373, 675.

Cane, H. V., I. G. Richardson, T. T. von Rosenvinge, and G. Wibberenz (1994), Cosmic ray decreases and shock structure: A multi-spacecraft study, J. Geophys. Res., 99, 21,429.

Cane, H. V., I. G. Richardson, and T. T. von Rosenvinge (1996), Cosmic ray decreases: 1964-1994, J. Geophys. Res., 101, 21,561.

Cane, H. V., W. C. Erickson, and N. P. Prestage (2002), Solar flares, type III radio bursts, coronal mass ejections and energetic particles, J. Geophys. Res., 107(A10), 1315, doi:10.1029/2001JA000320.

Cane, H. V., T. T. von Rosenvinge, C. M. S. Cohen, and R. A. Mewaldt (2003), Two components in major solar particle events, Geophys. Res. Lett., 30(12), 8017, doi:10.1029/2002GL016580.

Desai, M. I., G. M. Mason, J. R. Dwyer, J. E. Mazur, R. E. Gold, S. M. Krimigis, C. W. Smith, and R. M. Skoug (2003), Evidence for a suprathermal seed population of heavy ions accelerated by interplanetary shocks near 1 AU, Astrophys. J., 588, 1149.

Feldman, W. C., J. R. Ashbridge, S. J. Bame, J. T. Gosling, and R. D. Zwickl (1983), Electron heating at interplanetary shocks, in Solar Wind Five, edited by M. Neugebauer, NASA Conf. Publ., CP-2280, 403.

Fludra, A., and J. T. Schmelz (1999), The absolute coronal abundances of sulfur, calcium, and iron from Yohkoh-BCS flare spectra, Astron. Astrophys., 348, 286.

Kallenrode, M.-B. (1996), A statistical study of $5 \mathrm{MeV}$ proton events at transient interplanetary shocks, J. Geophys. Res., 101, 24,393.

Klecker, B., M. Scholer, D. Hovestadt, G. Gloeckler, and F. M. Ipavich (1981), Spectral and compositional variations of low energy ions during an energetic storm particle event, Astrophys. J., 251, 393.

Lin, R. P. (1970), The emission and propagation of $\sim 40 \mathrm{keV}$ solar flare electrons II The electron emission structure of large active regions, Solar Phys., 15, 453.

Mason, G. M., J. E. Mazur, and J. R. Dwyer (1999), ${ }^{3}$ He Enhancements in large solar energetic particle events, Astrophys. J., 525, L133.

McComas, D. J., S. J. Bame, P. Barker, W. C. Feldman, J. L. Phillips, P. Riley, and J. W. Griffee (1998), Solar Wind Electron Proton Alpha Monitor (SWEPAM) for the Advanced Composition Explorer, Space Sci. Rev., 86, 563.

Reames, D. V. (1998), Solar energetic particles: Sampling coronal abundances, Space Sci. Rev., 85, 327.

Reames, D. V. (2002), Magnetic topology of impulsive and gradual solar energetic particle events, Astrophys. J., 571, L63.

Reames, D. V., and C. K. Ng (2004), Heavy-element abundances in solar energetic particle events, Astrophys. J., 610, 510.
Reames, D. V., B. R. Dennis, R. G. Stone, and R. P. Lin (1988), X-ray and radio properties of solar ${ }^{3} \mathrm{He}$-rich events, Astrophys. J., 327, 998.

Reames, D. V., H. V. Cane, and T. T. von Rosenvinge (1990), Energetic particle abundances in solar electron events, Astrophys. J., 357, 259.

Richardson, I. G., and H. V. Cane (1996), Particle flows observed in ejecta during solar event onsets and their implication for the magnetic field topology, J. Geophys. Res., 101, 27,521.

Richardson, I. G., H. V. Cane, and T. T. von Rosenvinge (1991), Prompt arrival of solar energetic particles from far eastern events: The role of large-scale interplanetary magnetic field structure, J. Geophys. Res., 96, 7853

Sanderson, T. R., R. Reinhard, P. van Nes, and K.-P. Wenzel (1985), Observations of three-dimensional anisotropies of 35- to $1000-\mathrm{keV}$ protons associated with interplanetary shocks, J. Geophys. Res., 90, 19.

Smith, C. W., J. L'Heureux, N. F. Ness, M. H. Acuña, L. F. Burlaga, and J. Scheifele (1998), The ACE magnetic fields experiment, Space Sci. Rev., 86, 613.

Stone, E. C., et al. (1998), The Solar Isotope Spectrometer for the Advanced Composition Explorer, Space Sci. Rev., 86, 357.

Tsurutani, B. T., and R. P. Lin (1985), Acceleration of greater than $47 \mathrm{keV}$ ions and greater than $2 \mathrm{keV}$ electrons by interplanetary shocks at $1 \mathrm{AU}$, J. Geophys. Res., 90, 1.

Tylka, A. J., C. M. S. Cohen, W. F. Dietrich, M. A. Lee, C. G. Maclennan, R. A. Mewaldt, C. K. Ng, and D. V. Reames (2005), Shock geometry, seed populations, and the origin of variable elemental composition at high energies in large gradual solar particle events, Astrophys. J., 625, 474.

van Nes, P., R. Reinhard, T. R. Sanderson, K.-P. Wenzel, and R. D. Zwickl (1984), The energy spectrum of $35-$ to $1600-\mathrm{keV}$ protons associated with interplanetary shocks, J. Geophys. Res., 89, 2122.

von Rosenvinge, T. T., et al. (1995), The Energetic Particles: Acceleration, Composition, and Transport (EPACT) investigation on the WIND spacecraft, Sp. Sci. Rev., 71, 155.

von Rosenvinge, T. T., et al. (2001), Time variations in elemental abundances in solar energetic particle events, in Solar and Galactic Composition, edited by R. F. Wimmer-Schweingruber, pp. 343-348, Am. Inst. of Phys., Melville, N. Y.

H. V. Cane and T. T. von Rosenvinge, Astroparticle Physics Laboratory, Code 661, NASA Goddard Space Flight Center, Greenbelt, MD 20771, USA. (hilary.cane@utas.edu.au; tycho@milkyway.gsfc.nasa.gov)

C. M. S. Cohen and R. A. Mewaldt, Space Radiation Laboratory, California Institute of Technology, Pasadena, CA 91125, USA. (cohen@ srl.caltech.edu; rmewaldt@srl.caltech.edu) 